\title{
Parallel scanning of auditory and visual information
}

\author{
DAVID BURROWS and BARRY A. SOLOMON \\ State University of New York, College at Brockport, Brockport, New York 14420
}

\begin{abstract}
Two experiments involving memory retrieval of auditorily and visually presented materials were performed. In Experiment I, subjects were presented with memory sets of 1, 2, or 4 stimuli and then with a test item to be classified as belonging or not belonging to the memory set. In Condition 1, each memory stimulus was a single, auditorily presented letter. In Condition 2 , each memory stimulus was a visually presented letter. In Conditions 3 and 4, each memory stimulus was a pair of letters, one presented visually and the other auditorily. Mean reaction time (RT) for the classification task increased as a function of number of memory stimuli at equal rates for all four conditions. This was interpreted as evidence for a parallel scanning process in Conditions 3 and 4 where the auditory item and visual item of each memory stimulus pair can be scanned simultaneously. Experiment II compared memory retrieval for a simultaneous condition in which auditory and visual memory items were presented as pairs with a sequential condition in which mixed auditory-visual memory sets were presented one item at a time. RTs were shorter for the simultaneous condition. This was interpreted as evidence that parallel scanning may depend upon memory input parameters.
\end{abstract}

These two studies investigate the problem of how people retrieve information from memory. In particular, they are concerned with whether people are able to retrieve information about two or more different sets of memory information at the same time. If such simultaneous retrieval is possible, then an assumption of parallel processing can be built into models of the retrieval process, making possible theories of considerable power.

Experiment I was an attempt to demonstrate simultaneous retrieval of auditorily and visually presented information from short-term memory.

Sternberg $(1966,1969)$ has argued that retrieval from short-term memory involves a serial scan of the items in memory. He based this argument on the results of a series of experiments in which subjects were required to decide whether or not a test item was included in a short list of previously memorized items. Typically, the reaction time (RT) for the required two-choice response is a linearly increasing function of the number of items in the memory set. Each additional memory item seems to add a constant to the time required for the decision. This suggests that, before a decision is made, subject compares the test item with each memory item. Each comparison requires (on the average) one unit of time. The linearly increasing function reflects the increasing number of comparisons required when additional memory items are involved. The slope of the function is a measure of the rate of retrieval.

If large amounts of memory information are involved in a retrieval situation, a simple serial scan would be inefficient. Any general theory of how humans retrieve information from their memories must almost certainly involve processes more complex than simple serial scans. One possible process, suggested by Burrows and Okada (1973), is that two or possibly more serial scans of different sets of memory information could be executed simultaneously under certain conditions. Burrows and
Okada required subjects in three separate conditions to decide whether a test item was an exact match of any memory item, (same condition), a synonym of any memory item, (synonym condition) or either an exact match or a synonym (same or synonym condition). For the same condition and for the synonym condition, RT increased linearly with the number of memory items, suggesting that serial scans were used in both cases. The same or synonym condition logically required that both a scan for exact matches and a scan for synonym matches be executed. If these two scans could not be executed simultaneously, then the slope for the same or synonym condition should have been equal to the sum of the slopes for the same and the synonym conditions. In fact, the slope for the same or synonym condition was less steep than the sum of the other two slopes and was roughly equal to their average. This argues for a parallel scan: subjects are able to scan for exact matches in parallel with scans for synonym matches.

Before the concept of parallel scanning can be used as part of a general retrieval theory, more of its characteristics must be known. One obvious question concerns the relationship that must exist between two sets of information if they are to be functionally separate and capable of being scanned in parallel. If two sets are functionally a single set, then presumably they can be checked only with one, extensive serial scan. In the Burrows and Okada study, the two sets of information were two alternative representations, one semantic, the other formal, of the same items. The present Experiment I attempted to discover if parallel scanning of two sets of information is possible when the two memory sets represent completely independent items. The technique was to have subjects memorize a set of auditorily presented items and a different set of visually presented items and then test for parallel scanning. Recent evidence suggests that auditorily and visually presented stimuli are maintained via different 
memory codes in short-term memory (Murdock, 1966, 1967, 1968; Madsen, Rollins, \& Senf, 1970). If visual and auditory codes are separate, then parallel scanning of them might be possible.

The experiment had four conditions. In Condition 1, a set of memory items was presented auditorily and followed by either a visual or auditory test item to be classified as belonging or not belonging to the memory set. Condition 2 was equivalent to Condition 1 except that the memory items were presented visually. In Condition 3, each memory stimulus consisted of two simultaneously presented items, one auditory and one visual. A single auditory or visual test item was then presented, to be classified as identical to one of the items in the memory stimulus set or not identical to any memory item. Condition 4 was equivalent to Condition 3, except that if the test item was present in the set of memory stimuli, it was in the same modality for memory set and test item presentation. The parallel scanning hypothesis is tested by calculating mean RT as a function of number of memory stimuli for each condition. The slope of the resulting function for Condition 1 is a measure of the mean time for comparing a test item to an auditorily presented memory item. The slope for Condition 2 is a measure of comparison times for visually presented items. In Condition 3 , each memory stimulus required one auditory memory comparison and one visual memory comparison. If these comparisons cannot be executed in parallel, then the time for checking each memory stimulus would be the sum of the times for checking a Condition 1 and a Condition 2 memory stimulus. The slope of RT vs. number of memory stimuli in Condition 3 would be the sum of the slopes for Conditions 1 and 2 . If complete parallel scanning is possible, then the time for a Condition 3 comparison would be equivalent to the slower of the Condition 1 and Condition 2 comparisons. The slope for Condition 3 would be equal to the steeper of the Condition 1 and 2 slopes, but less than their sum.

If the results suggested that parallel scanning is not possible, then Condition 4 would test the hypothesis that a selective serial scan is possible when two distinct memory sets are presented. Since the test item, if presented at all, was presented in the same modality as in the memory set, the subject might be able to scan selectively one of the two memory sets, even it ne cannot scan both in parallel. In that case, the slopes for Condition 4 would be similar to the slopes for Condition 1 or Condition 2, depending upon the modality of the test item. If selective scanning were not possible, Condition 4 would be equivalent to Condition 3 . If the comparisons of Conditions 1,2 , and 3 support the hypothesis of parallel scanning, then Condition 4 should be a case where parallel scanning is possible and Conditions 3 and 4 should yield equivalent
results.

\section{EXPERIMENT I}

\section{Method}

Subjects. The subjects were 76 undergraduate men and women enrolled in a general psychology course at the State University College at Brockport. Participation was rewarded by adding one percentage point to each subject's final course grade.

Apparatus. Auditory stimuli were recorded in a male voice with a Wollensak tape recorder Model 1520 and presented binaurally via Wollensak headphones Model A-0483. Visual stimuli were presented on $35 \mathrm{~mm}$ slides by a Kodak Carousel Projector. The slides were made by typing the stimuli on paper with an IBM Selectric typewriter, transferring them to transparencies and mounting the transparencies in slide frames. The slides were projected on a wall approximately $2 \mathrm{~m}$ from the subject. Synchronization of auditory and visual stimuli was controlled by a Uher diapilot Model F422. The diapilot read prerecorded signals from the auditory tape and closed a relay which advanced the slide projector. Reaction tímes were measured to the nearest $.01 \mathrm{sec}$ by a Standard electric timer which was started by the diapilot. ${ }^{1}$ The subject signaled his response by pressing one of two push buttons mounted in a horizontal orientation. A set of two neon lights was used to signal the experimenter the identity of the subject's response.

Procedure. Each trial began with the presentation of the word "start" as a warning signal. This was followed by a memory set of 1,2 or 4 stimuli, presented sequentially at a rate of 2.5 $\mathrm{sec} / \mathrm{stimulus}$. The memory set was followed by the warning word "ready" and then the test item. The subject was instructed to decide as quickly as possible whether the test item matched any memory set item and press the key designated (for that subject) as "yes" if a match was found and the "no" key if no match was found. The next trial began $4 \mathrm{sec}$ after presentation of the test item. In Condition 1, the word "start" was presented auditorily, and each memory stimulus was an auditorily presented single letter. Half of the test items were presented visually and half auditorily. In Condition 2, the word "start" was visual, and each memory stimulus was a single visual letter. Half of the test items were visual and half auditory. In Conditions 3 and 4 , the word "start" was presented both visually and auditorily, and each memory stimulus was a simultaneously presented pair of letters, one presented visually and one auditorily. The test item was a single letter, presented visually on half of the trials and auditorily on the other half of the trials. The word "ready," the warning signal for the test item, was presented in the same modality as the test item for all four conditions.

Each subject served in one of the four conditions. After a set of 10 practice trials, the subject worked through 72 test trials. The 72 trials consisted of 24 at each of the three sizes of memory stimulus set, (1, 2 or 4 stimuli). For each of the 24 trials at a particular set size, 12 test items were auditory and 12 visual, 6 of each requiring a "yes" response and the remaining 6 requiring a "no" response. For Condition 3,3 of the 6 positive test items in each modality had been presented in the same modality in the memory set and the remaining 3 had been presented in the other modality. Subjects in Conditions 1,2 and 3 were instructed to respond positively to a test item if it was in the memory set at all, ignoring modality in making their decision. In Condition 4, all positive test items were presented in the same modality as test items and in the memory set. Subjects were encouraged to use this feature of the design to make the task easier in any way possible. For all conditions, each possible serial position in memory set presentation of a positive test item was sampled as evenly as possible.

Two sequences of 72 trials were constructed for each of the four conditions and randomly ordered. Ten of the 19 subjects in each condition were presented with one of the two resulting sets of trials, and the other nine were presented with the other set. 
Results

All trials on which errors occurred were discarded before any RT analyses were performed. The error rates for the four conditions were, respectively, $9 \%, 4 \%, 10 \%$ and $10 \%$. These are slightly higher than usually found with the Stemberg procedure, but are still low enough to justify discarding error data from the RT analysis.

The left-hand portion of Table 1 shows mean RT as a function of memory set size for each condition. Data for "yes" and "no" responses have been combined since set size affected RT's for both type of response equally. Since the main interest is in the slopes of the RT by set size functions, the usual procedure would be to fit straight lines to the data. However, inspection of the data indicates that the functions are negatively accelerated, approximating logarithmic functions. Since the Sternberg model predicts a linear function, this complicates any theoretical analysis, although negatively accelerated functions are not uncommon in this paradigm (Nickerson, 1972). Fortunately, Swanson and Briggs (1969) have developed a processing model which can account for such a finding. In the Swanson-Briggs analysis, memory retrieval essentially consists of resolving central processing uncertainty and RT is linearly related to central processing uncertainty. Under the usual conditions of the Sternberg paradigm, RT would be a $\log$ function of positive set size. ${ }^{2}$ Since it is not clear which model most clearly applies to the present data, slopes were calculated both for RT as a linear function of set size (Sternberg or linear model) and RT as a function of central processing uncertainty (Swanson-Briggs or log model), using least squares linear regression. For each model the slope value is a measure of information processing rate, and the logic of inferring parallel processing by comparing slopes applies to both models. All slope tests reported were done for both classes of tunctions.

As stated above, the hypothesis that subjects in the two-modality conditions cannot process auditory and visual information at the same time predicts greater slope values for Conditions 3 or 4 than for 1 and 2 and that the slope for Condition 3 or Conition 4 should be equal to the sum of the slopes for Conditions 1 and 2 .

The slope values were not greater for Conditions 3 or 4 than for Conditions 1 or 2 , but were in fact smaller, for both the $\log$ and linear fits. An analysis of variance of the four fitted slopes indicated no differences among the four conditions, either for the linear model, $\mathrm{F}(3,4)<1$, or the $\log$ model $\mathrm{F}(3,4)<1$. This demonstrates that the rate of retrieval for pairs of items in Conditions 3 and 4 is equivalent to the rates for individual items in Conditions 1 and 2 . A second method for assessing the relative processing rates involved testing the slopes for Condition 3 or Condition 4 against the sum of the slopes for Conditions 1 and 2. If the
Table 1

Mean RT in Milliseconds as a Function of Set Size With Parameters for Best Fit to Log and Linear Models

\begin{tabular}{|c|c|c|c|c|c|c|c|}
\hline \multirow[b]{2}{*}{$\begin{array}{l}\text { Condi- } \\
\text { tion }\end{array}$} & \multicolumn{3}{|c|}{ Set Size } & \multicolumn{2}{|c|}{$\begin{array}{c}\text { Linear Model } \\
\text { RT }= \\
a+b \circ M \\
\end{array}$} & \multicolumn{2}{|c|}{$\begin{array}{c}\text { Log Model } \\
\mathrm{RT}= \\
\mathrm{a}+\mathrm{b} \cdot \mathrm{Hc}\end{array}$} \\
\hline & 1 & 2 & 4 & $\begin{array}{c}\text { Slope } \\
\text { (Msec/ } \\
\text { Stim- } \\
\text { ulus) }\end{array}$ & $\begin{array}{l}\text { Inter- } \\
\text { cept }\end{array}$ & $\begin{array}{c}\text { Slope } \\
\text { (Msec/ } \\
\text { Bit) }\end{array}$ & $\begin{array}{l}\text { Inter- } \\
\text { cept }\end{array}$ \\
\hline 1 & 633 & 716 & 854 & 73 & 563 & 221 & 418 \\
\hline 2 & 687 & 770 & 863 & 57 & 640 & 176 & 509 \\
\hline 3 & 715 & 865 & 905 & 57 & 696 & 190 & 543 \\
\hline 4 & 765 & 838 & 905 & 45 & 731 & 140 & 626 \\
\hline
\end{tabular}

Note-M = number of stimuli presented, $\mathrm{Hc}=$ central processing uncertainty associated with number of stimuli presented.

processing of visual and auditory information did not overlap at all, the values for Condition 3 or 4 should be equivalent to the sum of the values for 1 and 2 . To the extent that processing of auditory and visual information does overlap, the slope for Condition 3 or for Condition 4 should be less steep than the sum of the slopes for Conditions 1 and 2. Since the earlier analysis indicated no overall slope differences, the average of the slopes for Conditions 3 and 4 was taken as the best estimate of the slope in those complex conditions. This was compared against the sum of the slopes for Conditions 1 and 2 by a one sample $t$ test. (The logic of this test is described in Draper \& Smith, pp. 18-21). The slope for Conditions 3 and 4 was less than the sum of the slopes for Conditions 1 and 2 both for the linear model, $\mathrm{t}(4)=6.39, \mathrm{p}<.005$, and for the log model, $t(4)=8.87, p<.005$. This means that the processing rate per item is faster for the complex two-modality conditions than for the single modality conditions.

The data from Table 1 also suggest that the intercepts of the functions vary greatly, with the simple conditions having lower intercepts. Tests of the combined intercept for Conditions 3 and 4 against the combined intercept for Conditions 1 and 2 yielded significant differences for both the linear model $t(10)=6.61, p<.005$ and for the $\log$ model $t(10)=5.21, p<.005$. This can be taken as evidence that whatever processes contribute to the intercepts of the functions take longer to execute in the complex conditions than in the simple conditions (this intercept test is also described in Draper \& Smith, 1966).

\section{Discussion}

The results indicate that the processing rate per item for Conditions 3 and 4 is greater than the rate for 1 and 2. This means that at least to some extent, the subjects are able to process more items per unit time in the complex conditions than in the simple conditions. The processing of auditory items to some extent can be 
carried on at the same time as the processing of visual items, when auditory and visual items have been presented simultaneously. The fact that the actual numerical values of the processing rate are no greater for Conditions 3 and 4 than for 1 and 2 suggests that this overlap is complete; Auditory and visual items can be checked simultaneously. The results demonstrate that two independent sets of information can be checked at the same time, if they are different in modality.

The higher intercepts for Conditions 3 and 4 indicated in Table 1 suggest that this parallel processing does cost something: Since the memory information is in two different formats, it is necessary to encode the test item twice in Conditions 3 and 4; once as an auditory item and once as a visual item. In Conditions 1 and 2, all information is in one format, and only one encoding of the test item is necessary. According to the Sternberg model, encoding time for the test item contributes to the intercept value of the processing function. The results suggest that the complex encoding for Conditions 3 and 4 requires more time than the encoding for Conditions 1 and 2.

The present result conflicts with results reported by Burrows (1972) who found that a single, sequentially presented set of mixed auditory and visual items was not checked at a more rapid rate than pure visual or pure auditory sets. Procedural differences between this experiment and Burrows' (1972) experiment might be responsible for this difference: Having two streams of purely visual and purely auditory information, as in the present experiment, may make it easier to maintain the information in two separate channels that can be scanned in parallel. The Burrows (1972) procedure of randomly mixing auditory and visual items may require shifts in attention that make separation of channels difficult. Experiment II was designed to test this hypothesis by setting up a memory retrieval task that requires separation of auditorily and visually presented information and comparing the performance of a group that has simultaneous presentation of auditory-visual pairs, as in Experiment I, with a group which has items presented one at a time with random shifts between modalities.

\section{EXPERIMENT II}

In Experiment II, the subjects were instructed to respond positively to items only if they were presented in the same modality as test items and memory items. On $1 / 3$ of the trials, the test item was an item which had been presented in the memory set, but in the opposite modality. These were to be classified as negative test items. Subjects had to maintain information about how items were presented, as well as information about whether they had been presented at all. Since parallel scanning logically requires such separation, relative performance on this task should be an indicator of the ease with which parallel scanning can be executed given simultaneous or sequential presentation. Superior performance by the simultaneous group would then help to explain why there was evidence for parallel scanning in Experiment I, but not in the Burrows (1972) study.

\section{Method}

Subjects. The subjects were 16 men and women drawn from the same pool as in Experiment 1 . None had any experience in RT experiments.

Apparatus. The apparatus was the same as used in Experiment I.

Procedure. Each trial consisted of a set of 6 letters, 3 presented visually and 3 auditorily, followed by a "ready" signal and then a test letter. The subject was required to press the button designated "yes" if, and only if, the test item had been presented in the memory set in the same modality as on the test. There were three types of trials: "Yes" trials (described above), "intemal no" trials on which the test item had been presented in the memory set but not in the same modality, and "external no" trials on which the test item had not been presented in the memory set at all. Subjects had to respond positively to the "yes" trials and negatively to both types of "no" trials. Subjects indicated their yes-no decision by pressing one of the two buttons as quickly as possible. After a 4-sec pause, another trial began with the presentation of a new memory set. Each subject worked through 18 practice trials and 36 test trials, 12 yes, 12 internal no, and 12 external no. Each subject was assigned to either the "simultaneous" condition or the "sequential" condition. In the simultaneous condition, the memory set was presented as three auditory-visual pairs of letters, as in Conditions 3 and 4 of Experiment $I$, at a rate of $1.5 \mathrm{sec}$ per pair. In the sequential condition, the memory set was presented as six individual letters, three visual and three auditory, at a rate of $1.5 \mathrm{sec}$ per item. The sequencing of auditory and visual items was randomly permuted for each trial. During presentation of an auditory item, a blank slide was shown. In both conditons, the ready signal was the word "ready" presented in both modalities simultaneously. Two complete sets of 36 trials were generated, with the choice of trial type and letters making up the memory set randomized. Half of the test items were presented visually and half auditorily. Visual test items were used for 6 of the 12 trials of each type and auditory test items for the reamining 6 . For each set of 36 trials, the two conditions used equivalent trials; e.g., trial $n$ of a simultaneous set corresponded to trial $n$ of the same sequential set in terms of letters presented and their modality. Only the mode of presentation (simultaneous vs. sequential) was different. Four of the eight subjects in each condition worked through one set of materials, and the remaining four worked through the other set.

\section{Results}

Errors. Table 2 shows the mean proportion of errors for the two modes of presentation, with separate entries for each trial type. The error rates were high, probably reflecting both the difficulty of the tasks and the small number of trials combined with the inexperience of the subjects. The overall rates were nearly identical for the two groups: .18 for simultaneous and .19 for sequential. For purposes of analysis, the experiment was treated as a 2 by 3 factorial with the first factor mode of presentation and the second factor trial type. The total number of errors for each subject under each trial type was used as the basic datum. Analysis of variance showed that the two groups did not differ in error rate, 
$\mathrm{F}(1,14)<1$. There was a main effect of trial type, $F(2,28)=14.3, p<.01$. Inspection of Table 2 suggests that this effect is attributable to the much lower rate for the External no's as compared to the other two types. This effect is equivalent for the two modes of presentation, as evidenced by a lack of any Mode by Trials interaction: $F(2,28)<1$.

Reaction Times. Table 3 shows mean RTs for correct responses for the two modes of presentation with separate entries for each trial type. There is a large difference between the modes of presentation, with the RTs for the sequential group $496 \mathrm{msec}$ longer than for the simultaneous group. A 2 by 3 analysis of variance was performed with each subject's mean RT for correct responses for each trial type as the basic datum. The difference between the two modes of presentation was significant: $F(1,14)=5.45, p<.05$. There was a main effect of trial type, $F(2,28)=9.77, p<.01$ but no interaction between mode of presentation and trial type: $F(2,28)=2.95$, N.S. External nos were responded to more rapidly than the other two trial types for both modes of presentation.

\section{Discussion}

Subjects are clearly able to respond more rapidly under the simultaneous presentation condition when required to separate auditory and visual items. This relatively greater ability to segregate the two sets of information makes possible the parallel scanning evidenced by the results of Experiment I. It is essential that two sets of information be separated before parallel scanning can occur. When items cannot be separated, a single serial scan must be carried out. The differences in separability are consistent with the evidence that parallel scanning is possible with simultaneous presentation but not with sequential presentation. In retrospect, this is not surprising. The simul taneous condition provides the opportunity for two, modality specific, continuous records of the auditory and visual stimuli, unbroken by switches to another modality. In the sequential condition, the record for each modality must contain "dead time" whenever a switch to the other mode of presentation takes place. This seems logically more difficult to scan, and the results confirm that it is.

These experiments demonstrate two things: (1) Parallel scans can improve the efficiency of memory retrieval when the prerequisite separation of information

Table 2

Mean Proportion of Errors for Each Mode of Presentation and Trial Type Combination: Experiment II

\begin{tabular}{lcccc}
\hline & \multicolumn{4}{c}{ Trial Type } \\
\cline { 2 - 5 } $\begin{array}{c}\text { Mode of } \\
\text { Presentation }\end{array}$ & Yes & $\begin{array}{c}\text { Internal } \\
\text { No }\end{array}$ & $\begin{array}{c}\text { External } \\
\text { No }\end{array}$ & Mean \\
\hline Simultaneous & .26 & .24 & .05 & .18 \\
Sequential & .32 & .24 & .01 & .19 \\
Mean & .29 & .24 & .03 & .18 \\
\hline
\end{tabular}

Table 3

Mean RT in Milliseconds for Correct Responses for Each Mode of Presentation and Trial Type Combination: Experiment II

\begin{tabular}{lcccc}
\hline & \multicolumn{4}{c}{ Trial Type } \\
\cline { 2 - 5 } $\begin{array}{c}\text { Mode of } \\
\text { Presentation }\end{array}$ & Yes & $\begin{array}{c}\text { Internal } \\
\text { No }\end{array}$ & $\begin{array}{c}\text { External } \\
\text { No }\end{array}$ & Mean \\
\hline Simultaneous & 1084 & 1112 & 860 & 1018 \\
Sequential & 1601 & 1713 & 1203 & 1506 \\
Mean & 1343 & 1413 & 1032 & 1262 \\
\hline
\end{tabular}

exists; (2) Input conditions can affect this separability in important ways.

One further implication is that retrieval should be more efficient if a set of memorized items belongs to several discriminable classes than if the set is homogeneous. In the limiting case, if each remembered item belongs to a separate "channel" and all channels can be checked in parallel, an extremely efficient retrieval could result. Demonstrating the possibility of such a retrieval system may prove to be a rewarding direction for further research.

\section{REFERENCES}

Burrows, D. Modality effects in retrieval of information from short-term memory. Perception \& Psychophysics, 1972, 11. 365-372.

Burrows, D., \& Okada, R. Parallel scanning of semantic and formal information. Joumal of Experimental Psychology, 1973, 97, 254-257.

Draper, M. R, \& Smith, H. Applied regression analysis. New York: Wiley, 1966.

Madsen, M. C., Rollins, H. A., \& Senf, G. M. Variables affecting immediate memory for bisensory stimuli: Eye-ear analogue studies of dichotic listening. Journal of Experimental Psychology, 1970, 83 (3, Pt. 2).

Murdock. B. B., Jr. Visual and auditory stores in snorteress memory. Quarterly Joumal of Experimental Psychology, 1966, 18, 206-211.

Murdock, B. B., Jr. Visual and auditory stores in short-term memory. Acta Psy chologica, 1967, 27, 316-324.

Murdock, B. B., Jr. Modality effects in short-term memory: Storage or retrieval? Journal of Experimental Psychology, $1968,77,79-86$.

Nickerson, $R$. S. Binary-classification reaction time: A review of some studies of information-processing capabilities. Psychonomic Monograph Supplements, 1972, 4, 275-318.

Sternberg, S. High-speed scanning in human memory. Science, $1966,153,652-654$

Stermberg, S. Memory-scanning: Mental processes revealed by reaction time experiments. American Scientist, 1969, 57, 421-457.

Swanson, J. M., \& Briggs, G. E. Information processing as a function of speed versus accuracy. Journal of Experimental Psy chology, 1969, 81, 223-229.

\section{NOTES}

1. This procedure resulted in the addition of a constant for each subject's RT for both auditory and visual stimuli, since visual stimuli were not actually presented until the projector changed slides and auditory stimuli were synchronized with the actual appearance of each slide. This delay was determined to be about .91 seconds when measured with a photocell relay. All data reported in the paper have been corrected by subtracting this constant.

2. See Swanson and Briggs (1969) for details of how central processing uncertainity is measured. 